DOI: https://doi.org/10.34069/AI/2021.46.10.18

How to Cite:

Solaz-Portolés, J.J., Ferrer Roselló, T., \& Sanjosé López, V. (2021). Estudio de la influencia de la alineación semántica en la resolución de problemas verbales de estequiometría. Amazonia Investiga, 10(46), 184-190. https://doi.org/10.34069/AI/2021.46.10.18

\title{
Estudio de la influencia de la alineación semántica en la resolución de problemas verbales de estequiometría
}

\section{Study of the influence of semantic alignment on verbal stoichiometry problem solving}

Received: August 15, 2021

\section{Resumen}

Con este trabajo se quiere analizar el efecto de la denominada alienación semántica en la resolución de problemas verbales de estequiometría. Además, se pretende estudiar cómo la formación académica puede intervenir en este efecto. Para ello, se administraron al azar entre 160 estudiantes de educación secundaria de diversos niveles académicos (desde $9^{\circ}$ grado hasta $12^{\circ}$ grado) dos problemas de estequiometría con entidades discretas (moléculas). En uno de los problemas aparecían fracciones, y en el otro problema porcentajes. De las puntuaciones obtenidas en la resolución de los problemas y del ANOVA efectuado, se puede concluir que: a) los estudiantes consiguen un éxito significativamente mayor en el problema con fracciones, confirmándose el fenómeno de la alineación semántica; b) un mayor nivel académico mejora de forma significativa el rendimiento en la resolución de ambos problemas; y c) solo en los niveles académicos bajos $\left(9^{\circ}\right.$ y 10 grado $)$ hay diferencias significativas entre la puntuación del problema con fracciones y del problema con porcentajes.

Palabras clave: alineación semántica, educación secundaria, estequiometría, nivel académico, resolución de problemas.
Accepted: October 30, 2021

\author{
Written by: \\ Joan J. Solaz-Portolés ${ }^{11}$ \\ https://orcid.org/0000-0003-4690-6556 \\ Tania Ferrer Rosellón ${ }^{22}$ \\ https://orcid.org/0000-0002-8583-3567 \\ Vicente Sanjosé López ${ }^{73}$ \\ https://orcid.org/0000-0003-3806-1717
}

\begin{abstract}
The aim of this work is to analyze the effect of the so-called semantic alignment on stoichiometric word problem solving. In addition, the aim is to study how academic training can have an impact on this effect. For this purpose, two stoichiometry problems with discrete entities (molecules) were randomly administered to 160 secondary school students in different grade levels (from 9th grade to 12th grade). Fractions appeared in one of the problems and percentages in the other. From the scores obtained in solving the problems and from the ANOVA performed, it can be concluded that: a) students achieve significantly greater success in the problem with fractions, confirming the phenomenon of semantic alignment; b) a higher grade level significantly improves performance in solving both problems; and c) only in low grade levels (9th and 10th grade) there are significant differences between the score of the problem with fractions and the problem with percentages.
\end{abstract}

Keywords: grade level, problem solving, secondary school, semantic alignment, stoichiometry.

\footnotetext{
${ }^{71}$ Doctor en Ciencias Químicas y Profesor Titular de Didáctica de las Ciencias Experimentales. Universitat de València. España.

${ }^{72}$ Máster en Formación del Profesorado de Educación Secundaria. Estudiante. Universitat de València. España.

${ }^{73}$ Doctor en Ciencias Físicas y Catedrático de Didáctica de las Ciencias Experimentales. Universitat de València. España.
} 


\section{Introduction}

Un problema verbal es un problema cuya información viene dada mediante un texto que consta, normalmente, de varias frases. La resolución de problemas verbales es una práctica extendida en las aulas de ciencias y matemáticas de diferentes niveles educativos. Los problemas verbales suelen describir situaciones en las que se ven implicadas entidades (objetos, seres vivos, sistemas, etc.) que pueden ser modelados matemáticamente (Leiss, Schukajlow, Blum, Messner, \& Pekrun, 2010).

Los problemas verbales de estequiometría son muy usuales en la enseñanza y aprendizaje de la Química. Estos problemas son básicos en el currículum de Química e implican en su resolución tanto la comprensión de conceptos como de las distintas representaciones de la materia (Dahsah, \& Coll, 2008). Por esta razón, se han llevado a cabo muchos estudios sobre cómo se realiza su resolución (Fach, De Boer, \& Parchmann, 2007). Parece que una de las principales causas de las dificultades de los estudiantes en la resolución de problemas estequiométricos radica en el tránsito que se debe efectuar entre las distintas representaciones de las reacciones químicas: macroscópica, submicroscópica y simbólica (Ramnarain, \& Joseph, 2012). En este sentido, Davidowitz, Chittleborough y Murray (2010) han propuesto el uso de diagramas submicroscópicos para visualizar las entidades y proporciones presentes en las ecuaciones químicas.

Se ha tener presente que otra posible fuente de dificultades en la resolución de problemas estequiométricos puede estar en la utilización de determinados formatos en los números racionales (fracciones, decimales y porcentajes) que aparecen en los enunciados de los problemas. Sin embargo, esta faceta todavía no ha sido investigada. Justamente, el presente estudio inicia una línea de investigación que pretende abordar esta cuestión en la educación secundaria. $\mathrm{Su}$ primer objetivo fue, precisamente, analizar cómo influye la utilización de fracciones o porcentajes en la resolución de problemas verbales estequiométricos planteados con entidades submicroscópicas (moléculas).

A lo largo de la educación secundaria los y las estudiantes resuelven muchos problemas verbales en los distintos cursos y asignaturas, lo que les posibilita asimilar conocimientos y estrategias de resolución de problemas. En este sentido, no es de extrañar que se haya encontrado que a mayor nivel académico de estudiantes de secundaria mayor éxito en la resolución de problemas (Alabau, Solaz-Portolés, \& Sanjosé, 2020), y que este efecto sea más notorio cuanto mayor complejidad estructural tienen los problemas (García, Sanjosé, \& Solaz-Portolés, 2015). Por ello, el segundo objetivo del estudio fue investigar el efecto del nivel académico de los estudiantes de secundaria sobre el uso de fracciones o porcentajes en la resolución de problemas verbales de estequiometría con moléculas.

\section{Marco Teórico e hipótesis}

Para explicar los procesos que se llevan a cabo los estudiantes durante la resolución de los problemas verbales se han propuesto diferentes modelos de resolución de problemas. Uno de los modelos más citados en la bibliografía es el Nathan, Kintsch y Young (1992). Estos autores plantean en su investigación un modelo de resolución de problemas algebraicos que postula que los estudiantes durante la resolución de un problema verbal elaboran, en primer lugar, una representación proposicional del texto del problema, la base de texto. A continuación, construyen una representación cualitativa de carácter personal a partir de la base de texto, y de los conocimientos del estudiante, denominada modelo de la situación. Finalmente, llegan a una representación cuantitativa, el modelo del problema, que contiene las relaciones formales y recoge la estructura o esquema del problema. Para llegar a esta última representación se requiere que el estudiante ya tenga experiencia previa en resolución de problemas y disponga en su memoria a largo plazo de estructuras o esquemas de problema (esto es, modelos matemáticos) que pueda activar a partir del modelo de la situación (Dixon, 2005; Waldmann, 2007). Sin embargo, si se produce una disonancia entre el modelo de la situación (cualitativo) y el modelo de problema (cuantitativo), Coquin-Viennot y Moreau (2007) han demostrado que la activación de las representaciones formales se dificulta y se reduce el éxito en la resolución del problema.

Martin y Bassok (2005) propusieron que la modelización matemática de un problema verbal se basa en la denominada alineación semántica, es decir, en la alineación analógica entre la relación semántica extraída a partir de las entidades presentes en el enunciado del problema y la relación matemática entre los operandos (o argumentos) de una operación matemática determinada. Así, por ejemplo, si tuviéramos un 
problema de distribución de flores entre jarrones, la relación semántica de "contención" de las flores en los jarrones se trasladaría a una relación matemática cuya operación sería la división.

Diferentes trabajos han constatado una alineación semántica en problemas verbales que contienen números racionales con diferentes formatos (fracción, decimal o porcentaje). Se ha observado que los estudiantes tienden a establecer una alineación entre entidades discretas (bolas, por ejemplo) y fracciones, y entre entidades continuas (agua, por ejemplo) y números decimales (DeWolf, Bassok, \& Holyoak, 2015; Fisher, \& Bassok, 2009). Esta misma alineación ha sido encontrada en los libros de texto (DeWolf, Rapp, Bassok, \& Holyoak, 2014), y en estudiantes de diferentes entornos socioculturales (Lee, DeWolf, Bassok, \& Holyoak, 2016). Además, en el trabajo de Gray, DeWolf, Bassok y Holyoak (2018) se evidenció que los porcentajes producen mayoritariamente los mismos efectos que los números decimales, esto es, tienden a ser alineados con entidades continuas.

Por otra parte, diversos estudios han analizado la evolución de conocimientos, capacidades y estrategias en la resolución de problemas en el período que corresponde a la educación secundaria. Byrnes (2003) constató el avance en conocimientos, razonamiento, capacidad de la memoria de trabajo y estrategias metacognitivas con la edad. Xin y Zhang (2009) comprobaron, mediante la resolución de problemas analíticos y complejos, el desarrollo durante la adolescencia de los componentes necesarios para la resolución de problemas. Molnár, Greiff y Csapó (2013) mostraron un aumento de las habilidades en la educación secundaria en el razonamiento inductivo, dominio específico y resolución de problemas de dominio general en entornos complejos.

De acuerdo con todo este bagaje teórico, las hipótesis que se formulan en la presente investigación son:

H1.-Los y las estudiantes de educación secundaria que resuelvan un problema de estequiometría con moléculas cuyo enunciado contenga números racionales en forma de fracciones tendrán mayor éxito en su resolución que en el caso de que contenga dichos números racionales en forma de porcentaje.

H2.-El nivel académico de los y las estudiantes de educación secundaria mejorará el rendimiento de la resolución de problemas de estequiometría con moléculas y reducirá las diferencias entre los problemas donde aparecen fracciones $y$ porcentajes.

\section{Metodología}

En el estudio han participado 160 estudiantes españoles de dos institutos públicos de educación secundaria valencianos de distintos grupos y niveles académicos. De ellos, 41 cursan $9^{\circ}$ grado ( $3^{\circ}$ de Educación Secundaria Obligatoria, ESO, en el sistema educativo español, edad 14 años), otros 46 cursan $10^{\circ}$ grado ( $4^{\circ}$ de ESO, edad 15 años), 44 estudian $11^{\circ}$ grado $\left(1^{\circ}\right.$ de Bachillerato en el sistema educativo español, edad 16 años) y, por último, 29 están en el $12^{\circ}$ grado $\left(2^{\circ}\right.$ de Bachillerato). Todos los participantes de los grados $11^{\circ}$ y $12^{\circ}$ (estudiantes de Bachillerato) siguen un itinerario académico científico o técnico.

Los participantes en el estudio no parecen tener, a priori, características especiales que los diferencien de otros grupos de los respectivos cursos. No obstante, hemos de indicar que no se realizó muestreo aleatorio alguno, ya que se trató de una muestra de conveniencia. Por ello, los resultados no pueden ser extrapolados a toda la población estudiantil de esos cursos, esto es, no hay garantías de validez externa.

Para llevar a cabo la presente investigación se elaboraron dos problemas. El enunciado de ambos problemas es prácticamente idéntico (se basa en la reacción de formación de amoníaco a partir de gas nitrógeno y gas hidrógeno), diferenciándose únicamente en la forma matemática en que proporcionan los datos. En un caso se proporcionaron en forma de fracción (Problema 1) y en el otro caso en forma de porcentaje (Problema 2). A continuación, se muestran los enunciados:

$\mathrm{P}-1$. El gas nitrógeno $\left(\mathrm{N}_{2}\right)$ reacciona con el gas hidrógeno $\left(\mathrm{H}_{2}\right)$ en determinadas condiciones produciendo amoníaco $\left(\mathrm{NH}_{3}\right)$. Se sabe que reaccionan en la proporción $1 / 3$ (una molécula de nitrógeno por cada tres de hidrógeno). ¿Cuántas moléculas de nitrógeno se necesitan para reaccionar con 99 moléculas de hidrógeno? ¿Cuántas moléculas de amoníaco se obtendrán tras la reacción?

$\mathrm{P}-2$. El gas nitrógeno $\left(\mathrm{N}_{2}\right)$ reacciona con el gas hidrógeno $\left(\mathrm{H}_{2}\right)$ en determinadas condiciones produciendo amoníaco $\left(\mathrm{NH}_{3}\right)$. Se sabe que, cuando reaccionan, el $25 \%$ de las moléculas son de nitrógeno y el $75 \%$ son de hidrógeno. ¿Cuántas moléculas de nitrógeno se necesitan 


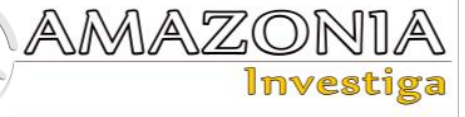

para reaccionar con 99 moléculas de hidrógeno? ¿Cuántas moléculas de amoníaco se obtendrán tras la reacción?

La resolución de los problemas se llevó a cabo durante el horario de clase normal, cada grupo en su aula y profesor. Se distribuyó al azar entre los estudiantes uno de los dos problemas. Dispusieron de un tiempo máximo de 25 minutos para su resolución. Una vez recogidas todas las muestras, se aplicó para su evaluación a todos los problemas una rúbrica que ya se ha utilizado en otras investigaciones (García et al., 2015; SolazPortolés \& Caballer, 2015), donde se atienden aspectos tales como la elaboración de un diagrama como base de la resolución, el planteamiento correcto de las relaciones de proporcionalidad directa entre las variables implicadas, si las operaciones efectuadas son correctas, si se despeja la variable de forma correcta, así como si se presentan de forma clara y correcta las respuestas. La calificación máxima para cada problema es de 10 puntos. Para comprobar que el sistema de puntuación podía interpretarse de forma fiable un total de 50 estudiantes fueron evaluados por dos correctores distintos (un autor de este trabajo y otro corrector externo profesor universitario). Posteriormente, se calculó el coeficiente de correlación de Pearson entre las puntuaciones otorgadas en cada uno de los cinco puntos de la rúbrica. Estos coeficientes oscilaron entre 0.81 y 0.92 , con una media de 0.88. En consecuencia, puede considerarse que el procedimiento de evaluación es suficientemente objetivo.

\section{Resultados y discusión}

La Tabla 1 recoge las puntuaciones promedio (y las desviaciones estándar entre paréntesis) en ambos problemas en cada uno de los niveles académicos.

Tabla 1.

Puntuaciones promedio en el Problema 1 y Problema 2 en función del nivel académico de los participantes. La desviación estándar se proporciona entre paréntesis.

\begin{tabular}{lll}
\hline Nivel académico & Problema 1 (Fracción) & Problema 2 (Porcentaje) \\
\hline $9^{\circ}$ grado & $5.95(4.01)$ & $4.00(3.83)$ \\
$10^{\circ}$ grado & $7.33(3.16)$ & $5.27(3.95)$ \\
$11^{\circ}$ grado & $7.60(2.72)$ & $7.16(2.50)$ \\
$12^{\circ}$ grado & $7.78(3.14)$ & $7.73(3.49)$ \\
\hline
\end{tabular}

Fuente: Elaboración propia

El test de Shapiro-Wilk aplicado a las puntuaciones del problema en cada nivel académico proporciona en todos los casos niveles de significación p superiores a 0.05 . En consecuencia, en todos los niveles se puede rechazar la hipótesis nula y puede considerarse que todas las puntuaciones siguen una distribución normal.

Como se puede observar en la Tabla 1, hay una clara correspondencia entre el éxito resolutivo de ambos problemas y el nivel académico, siendo la puntuación más elevada para los niveles académicos más altos. A su vez, también podemos comprobar que la resolución del Problema 2 (con porcentajes) ha obtenido menor puntuación que la del problema 1 , siendo mayor las diferencias de puntuación entre ambos problemas en los niveles académicos inferiores.

Se llevó a cabo un análisis de varianza (ANOVA) tomando como variables intersujetos el tipo de problema (con dos niveles: problema con fracción y problema con porcentaje) y el nivel académico (con cuatro niveles: $9^{\circ}, 10^{\circ}, 11^{\circ}$ y $12^{\circ}$ grado); y como variable dependiente la puntuación en el problema. Los resultados de este ANOVA revelan que: a) la variable tipo de problema genera diferencias estadísticamente significativas en la puntuación obtenida por los estudiantes (aunque con un tamaño del efecto pequeño), $\mathrm{F}(1,152)=3.90, \mathrm{p}<.05, \eta^{2}=0.025 ; \mathrm{y}$ b) la variable nivel académico influye significativamente en la puntuación del problema (con un tamaño del efecto medio-alto), F $(3,152)$ $=4.61, \mathrm{p}<.01, \eta^{2}=0.083$. Sin embargo, la interacción entre ambas variables no produce efectos significativos. Es decir, aunque se observa que las diferencias de puntuación entre ambos problemas no son las mismas en los distintos niveles académicos (muy poca diferencia en el nivel académico más alto y gran diferencia en el más bajo), estas diferencias no son estadísticamente significativas.

Un análisis post hoc mediante el test de Scheffé mostró diferencias significativas entre las puntuaciones del problema con fracción y el problema con porcentaje en $9^{\circ}$ grado $(\mathrm{p}<.01)$ y en $10^{\circ}$ grado $(\mathrm{p}<.01)$. También pone de manifiesto 
que en el problema con fracción solo las puntuaciones de los estudiantes de $12^{\circ}$ grado son significativamente mejores que las de $9^{\circ}$ grado $(p<.05)$. En cambio, en el problema con porcentaje las puntuaciones de los estudiantes de $12^{\circ}$ grado difieren significativamente tanto de las de $9^{\circ}$ grado $(\mathrm{p}<.01)$, como de las de $10^{\circ}$ grado $(\mathrm{p}<.01)$.

Atendiendo a las diferencias significativas de éxito en la resolución del problema estequiométrico con entidades moleculares en función del formato numérico del enunciado, parece que en este tipo de problemas también se manifiesta el fenómeno de la alineación semántica. Esto es, dado que en el enunciado del problema aparecen entidades discretas (moléculas), el formato fracción ayuda más que el formato porcentaje al establecimiento del modelo del problema que permite su resolución. Estos resultados son coherentes con los trabajos de Fisher y Bassok (2009), Martin y Bassok (2005), y Gray et al. (2018).

La influencia significativa de la formación académica se deja notar sobre el rendimiento obtenido en la resolución del problema. De tal manera, que un mayor nivel académico supone una mayor puntuación en el problema de estequiometría en cualquiera de las dos versiones, y esto es acorde con anteriores investigaciones en la que se analizó el efecto del nivel académico sobre la resolución de problemas verbales de ciencias (García et al., 2015; Solaz-Portolés \& Caballer, 2015). No obstante, a tenor de los resultados obtenidos en el ANOVA, las puntuaciones del problema con fracción y del problema con porcentaje no llegan a ser significativamente distintas en todos los grupos de diferente nivel académico. De hecho, solo lo son en los niveles académicos más bajos.

\section{Conclusiones}

Antes de exponer las conclusiones que se derivan de este estudio, se hace necesario señalar sus limitaciones. La primera de ellas radica en la muestra, pequeña y seleccionada de forma no aleatoria. La segunda proviene de los instrumentos empleados. Se han utilizado solamente dos problemas (uno con fracción y otro con porcentajes) y solo figuraban entidades discretas (moléculas). Todas estas particularidades hacen restringir la generalización de los hallazgos. Por consiguiente, las conclusiones solamente pueden ser válidas stricto sensu para los y las estudiantes que han participado y los problemas verbales que se han utilizado.
En relación con la primera hipótesis planteada, los resultados la ratifican plenamente. Si acaso, puede añadirse que el éxito en el problema que contenga fracciones ha sido mayor que con porcentajes de forma estadísticamente significativa. Así pues, parece que se puede concluir que los y las estudiantes que resuelven un problema de estequiometría con moléculas donde aparecen fracciones tienen un éxito significativamente mayor que aquellos que resuelven el problema con porcentajes.

En la segunda hipótesis se hablaba de una doble influencia de nivel académico sobre los problemas verbales de estequiometría. Se ha observado que, en efecto, como apuntaba la hipótesis, el nivel académico hace mejorar de forma significativa el rendimiento en la resolución. Además, en dicha hipótesis se mencionaba que el nivel académico reduciría las diferencias entre la puntuación del problema con fracciones y la puntuación del problema con porcentajes. De hecho, se ha comprobado que en los niveles académicos bajos hay diferencias significativas entre la puntuación del problema con fracción y del problema con porcentajes. En los niveles académicos más altos continúa habiendo diferencias, pero no son estadísticamente significativas. Puede concluirse, pues, que el nivel académico mejora significativamente el rendimiento en la resolución de problemas de estequiometría con moléculas y consigue reducir las diferencias entre los problemas con fracciones y los problemas con porcentajes en los niveles académicos más altos.

Finalmente, señalar las implicaciones didácticas que se deducen del presente estudio. Se ha podido corroborar el efecto del fenómeno denominado alineación semántica en problemas verbales de estequiometría. Así, cuando se producen disonancias durante el proceso de modelización matemática provocadas por entidades presentes en el enunciado del problema y el formato de los datos que aparecen se dificulta notoriamente su resolución. Esta circunstancia debería tenerse en consideración por el profesorado de Química, sobre todo en los primeros cursos en los que se aborden los cálculos estequiométricos.

\section{Referencias Bibliográficas}

Alabau, J., Solaz-Portolés, J. J., \& Sanjosé, V. (2020) Relación entre creencias sobre resolución de problemas, creencias epistemológicas, nivel académico, sexo y desempeño en resolución de problemas: un 


\section{AMAZZOND杰}

estudio en educación secundaria. Revista Eureka sobre Enseñanza y Divulgación de las Ciencias, $17(1)$, 1102. https://doi.org/10.25267/Rev_Eureka_ensen _divulg_cienc.2020.v17.i1.1102

Byrnes, J. P. (2003). Cognitive development during adolescence. In G. R. Adams, \& M. D. Berzonsky (Eds.), Blackwell Handbook of Adolescence (pp. 227-246). Malden, MA: Blackwell Publishing.

Coquin-Viennot, D., \& Moreau, S. (2007). Arithmetic problems at school: When there is an apparent contradiction between the situation model and the problem model. British Journal of Educational Psychology, 77(1), 69-80. https://doi.org/10.1348/000709905X79121

Dahsah, C., \& Coll, R. K. (2008). Thai grade 10 and 11 students understanding of stoichiometry and related concepts. International Journal of Science and Mathematics Education, 6(3), 573-600. https://doi.org/10.1007/s10763-007-9072-0

Davidowitz, B., Chittleborough, G., \& Murray, E. (2010). Student-generated submicro diagrams: A useful tool for teaching and learning chemical equations and stoichiometry. Chemistry Education Research and Practice, 11(3), 154-164. https://doi.org/10.1039/C005464J

DeWolf, M., Bassok, M., \& Holyoak, K. J. (2015). Conceptual structure and the procedural affordances of rational numbers: Relational reasoning with fractions and decimals. Journal of Experimental Psychology: General, 144(1), 127. https://doi.org/10.1037/xge0000034

DeWolf, M., Rapp, M., Bassok, M., \& Holyoak, K. J. (2014). Semantic alignment of fractions and decimals with discrete versus continuous entities: A textbook analysis. In B. Bello, M. Guarini, M. McShane, \& B. Scassellati (Eds.), Proceedings of the 36th Annual Conference of the Cognitive Science Society (pp. 2133-2138). Austin, TX: Cognitive Science Society.

Dixon, J. A. (2005). Mathematical problem solving: the roles of exemplar, schema, and relational representations. In J. I. D. Campbell (Ed.), Handbook of mathematical cognition (pp. 379- 395). New York: Psychology Press.

Fach, M., De Boer, T., \& Parchmann, I. (2007). Results of an interview study as basis for the development of stepped supporting tools for stoichiometric problems. Chemistry Education Research and Practice, 8(1), 13-31. https://doi.org/10.1039/B6RP90017H
Fisher, K. J., \& Bassok, M. (2009). Analogical alignments in algebraic modeling. In B. Kokinov, K. J. Holyoak, \& D. Gentner (Eds.), Proceedings of the 2nd International Conference on Analogy (pp. 137-144). Sofia, Bulgaria: New Bulgarian University.

García, P., Sanjosé, V., \& Solaz-Portolés, J. J. (2015). Efectos de las características del problema, captación de su estructura y uso de analogías sobre el éxito de los estudiantes de secundaria en la resolución de problemas. Teoría de la Educación. Revista Interuniversitaria, 27(2), 221-244, http://dx.doi.org/10.14201/teoredu20152722 21244

Gray, M. E., DeWolf, M., Bassok, M., \& Holyoak, K. J. (2018). Dissociation between magnitude comparison and relation identification across different formats for rational numbers. Thinking \& Reasoning, 24(2), 179-197. https://doi.org/10.1080/13546783.2017.1367 327

Lee, H. S., DeWolf, M., Bassok, M., \& Holyoak, K. J. (2016). Conceptual and procedural distinctions between fractions and decimals: A cross-national comparison. Cognition, 147, 57-69. https://doi.org/10.1016/j.cognition.2015.11.0 05

Leiss, D., Schukajlow, S., Blum, W., Messner, R., \& Pekrun, R. (2010). The role of the situation model in mathematical modelling-Task analyses, student competencies, and teacher interventions. Journal for Mathematics Didactics, 31(1), 119-141. https://doi.org/10.1007/s13138010-0006-y

Martin, S. A., \& Bassok, M. (2005). Effects of semantic cues on mathematical modeling: Evidence from word-problem solving and equation construction tasks. Memory \& Cognition, 33(3), 471-478. https://doi.org/10.1007/10.3758/bf03193064

Molnár, G., Greiff, S., \& Csapó, B. (2013). Inductive reasoning, domain specific and complex problem solving: Relations and development. Thinking Skills and Creativity, 9 , 35-45. https://doi.org/10.1016/j.tsc.2013.03.002

Nathan, M. J., Kintsch, W., \& Young, E. (1992). A theory of algebra-word-problem comprehension and its implications for the design of learning environments. Cognition \& Instruction, 9(4), 329-389. https://doi.org/10.1207/s1532690xci0904_2

Ramnarain, U., \& Joseph, A. (2012). Learning difficulties experienced by grade 12 South African students in the chemical 
representation of phenomena. Chemistry Education Research and Practice, 13(4), 462-470. https://doi.org/10.1039/C2RP20071F

Solaz-Portolés, J. J., \& Caballer, A. (2015). Contexto, estructura y analogías en la resolución de problemas verbales algebraicos por maestros de primaria en formación. Revista Electrónica de Investigación Educativa, 17(3), 94-108. Recuperado de http://redie.uabc.mx/vol17no3/contenidosolaz-caballer.html
Waldmann, M. R. (2007). Combining versus analyzing multiple causes: How domain assumptions and task context affect integration rules. Cognitive Science, 31(2), 233-256.

https://doi.org/10.1080/15326900701221231

Xin, Z., \& Zhang, L. (2009). Cognitive holding power, fluid intelligence, and mathematical achievement as predictors of children's realistic problem solving. Learning and Individual Differences, 19(1), 124-129. https://doi.org/10.1016/j.lindif.2008.05.006 